\title{
Unimodular matrix and bernoulli map on text encryption algorithm using python
}

\author{
Samsul Arifin ${ }^{*}$, Indra Bayu Muktyas², Puguh Wahyu Prasetyo ${ }^{3}$, Abdul $^{2}$ \\ Azis Abdillah4 \\ ${ }^{1}$ Bina Nusantara University, Indonesia \\ ${ }^{2}$ Sekolah Tinggi Keguruan dan Ilmu Pendidikan Surya, Indonesia \\ ${ }^{3}$ Universitas Ahmad Dahlan, Indonesia \\ ${ }^{4}$ Politeknik Negeri Jakarta, Indonesia \\ 四 samsul.arifin@binus.edu
}

\section{Article Information}

Submitted Nov 29, 2021

Revised Dec 25, 2021

Accepted Dec 26, 2021

\section{Keywords}

Bernoulli Map; Hill cipher; Python; Unimodular Matrix.

\begin{abstract}
One of the encryption algorithms is the Hill Cipher. The square key matrix in the Hill Cipher method must have an inverse modulo. The unimodular matrix is one of the few matrices that must have an inverse. A unimodular matrix can be utilized as a key in the encryption process. This research aims to demonstrate that there is another approach to protect text message data. Symmetric cryptography is the sort of encryption utilized. A Bernoulli Map is used to create a unimodular matrix. To begin, the researchers use an identity matrix to generate a unimodular matrix. The Bernoulli Map series of real values in $(0,1)$ is translated to integers between 0 and 255. The numbers are then inserted into the unimodular matrix's top triangular entries. To acquire the full matrix as the key, the researchers utilize Elementary Row Operations. The data is then encrypted using modulo matrix multiplication.
\end{abstract}

\section{INTRODUCTION}

A computer network is a collection of computers connected to share resources, communicate, and access data. The goal of a computer network is to fulfill its goals, and any component of the network can request and deliver services. The client is the person who requests and receives the service, whereas the server is the person who delivers and sends the service. A client-server system is an architecture utilized in practically all computer network applications. Securing computer networks or data necessitates the use of appropriate procedures. In cryptography, this is a common strategy. Cryptography may be employed in a variety of ways to safeguard data. The Hill Cipher algorithm is one of the most often utilized methods. One of the implementations of Hill Chipper can be seen in (Nana \& Prasetyo, 2021). The Hill Cipher is a symmetric key cryptographic technique with various data encryption benefits. The key matrix is constructed using the new binomial coefficient Newton to prevent a non-invertible key matrix. Plaintext may employ picture or text media and uses the same key for encryption and description. The key to encryption and decryption in the Hill Cipher technique is an $\mathrm{m} \times \mathrm{m}$ sized matrix. Multiplication between matrices and inverses the matrix is the basic matrix theory employed in Hill Cipher. Hill Cipher is a cryptographic algorithm that uses modulo arithmetic. This cryptography approach encrypts and decrypts using a square matrix as the key. When the Hill Cipher algorithm is used to send and receive data, security is assumed to be assured. Thanks to this algorithm, users of data delivery services no longer 
have to be concerned about attackers attempting to compromise security systems and steal data (Siahaan, 2018).

Text messaging is quite significant in this decade. When text messages are sent from one person to another, protecting them is frequently done at the same time. This necessitates better text message security, both in sending and keeping them. Encrypting text messages is one method. The Hill cipher is a well-known encryption algorithm. The Hill cipher has seen several changes in recent years. However, this approach only works with $3 \times 3$ or $4 \times 4$ finite key matrices. When the key matrix size exceeds 4 , it is said that finding the inverse key is difficult or that finding the reversible key matrix would take a long time. A specific matrix, known as a unimodular matrix, can be used to overcome this problem (Arifin \& Muktyas, 2018). Using basic row operations, the researchers can create a unimodular matrix. More information on the unimodular matrix can be accessed (Guo \& Yang, 2013). It is unnecessary to utilize the complete matrix as a key. The researchers will create a unimodular matrix using a Bernoulli Map, which means the researchers will need fewer parameters.

Furthermore, using the Python 2.7.14 programming language, the proposed approach will be implemented in many text messages. Some of the reasons the researchers utilize Python are as follows. Python is a fairly simple language to learn, it is free as an open-source, and it is used widely by any programmer in our whole world. Hence, an implementation using Phyton is claimed to have a big impact on the community. This application is also really simple to obtain. Python is available on a variety of operating systems. Python has several applications across various skill sets and disciplines (Arifin et al., 2021; Oliphant, 2007).

Researches on communication security have always been an interesting topic. Recently, online activities cannot be neglected in the new normal era. That is why communication security is still being investigated to better and better. Some researches on communication security can be seen in (Muktyas et al., 2021; Nana \& Prasetyo, 2021). In Muktyas et al. (2021), the security on a message of image form has been developed.

Moreover, this research uses Python to implement a text message encryption technique using a unimodular matrix and a Bernoulli Map, as explained above. In Session 2, the researchers will talk about the research process and review the theory employed, followed by the Python code. Session 3 will cover the implementation of the algorithm and some analysis, and Session 4 will wrap up this work.

\section{METHODS}

Cryptography is the study of encryption techniques in which plaintext is converted to ciphertext by encrypting it with a secret key. Someone without a decryption key will be unable to solve this document. The ciphertext will be decrypted using an agreed-upon key, and the original data will be returned. In the not-too-distant future, the chances of someone without a decryption key recovering the original text are extremely slim. In classical cryptography, symmetric encryption is utilized, in which the decryption key is the same as the encryption key. Asymmetric encryption techniques are necessary for public-key cryptography when the decryption key is different from the encryption key. Because asymmetric encryption employs very big numbers, encryption, decryption, and key generation for asymmetric encryption techniques need more intense computer than symmetric encryption. Hill Cipher was designed by Lester S. Hill in 1929. This cryptographic approach was developed to build 
encryption that could not be cracked via frequency analysis. Because Hill Cipher employs matrix multiplication for encryption and decryption, it does not replace identical alphabets in plaintext with the same alphabet in the ciphertext. Because the text to be processed will be separated into blocks of a specific size, the Hill Encryption, a polyalphabetic cipher, may be classified as a block cipher. In the encryption and decryption process, each character in a block impacts the other characters, ensuring that the same character is not mapped to the same character.

Hill Cipher is one of the traditional cryptographic algorithms that cryptanalysts find extremely difficult to crack if they simply have access to the ciphertext file. However, this approach can be quickly solved if the cryptanalyst possesses a ciphertext file and a portion of the plaintext file. A known-plaintext attack is the name of this cryptanalysis approach. Modulo arithmetic to the matrix is the foundation of the Hill Cipher algorithm. Hill Cipher employs matrix multiplication and inverse matrices methods in its implementation. The matrix $\mathrm{n} x \mathrm{n}$, where $\mathrm{n}$ is the block size, is the key to Hill Cipher. Because the $\mathrm{K}-1$ matrix is the key required to decode, the $\mathrm{K}$ matrix that produces this key must be an invertible matrix with inverse K-1. As a result, the key must have an inverse (Siahaan, 2018; W, 2006).

In the year, Lester Hill found a way to encrypt a plaintext using the Linear Equation System. Before encrypting a plaintext, Hill Cipher divides it into blocks first. The ciphertext is generated by solving a System of Linear Equations consisting of $n$ equations and $n$ variables. The Linear Equation System can be solved using the matrix multiplication concept. Note that the concept of Hill Cipher belongs to asymmetrical cryptography, which means that the resulting key must have an inverse, which can be guaranteed by a unimodular matrix as the key matrix. The phrase "unimodular matrix" or "Nice Matrix" was coined by Hanson (1982). A square matrix A is called a unimodular matrix if it occurs $\operatorname{det}(\mathrm{A})=-1 \operatorname{or} \operatorname{det}(\mathrm{A})=1$. Some examples of unimodular machines are the upper triangle matrix and the lower triangle matrix, where all the main diagonal entries are 1 or -1 . This statement follows the following theorem (Anton \& Rorres, 2013; Muktyas et al., 2021).

(Theorem 1.)(Anton \& Rorres, 2013) Let $A$ is a triangle matrix.Then $\operatorname{det}(A)=a_{11} a_{22} \ldots a_{n n}$.

The following lemma describes the steps needed in generating a unimodular matrix. Note that Lemma 2 below will be the starting point for writing Python program code to build a unimodular matrix (Hanson, 1982; Arifin \& Muktyas, 2021).

Lemma 2. (Hanson, 1982) A unimodular matrix $A_{n}$ can be constructed in the following ways:

1. First, make a diagonal matrix with the diagonal entry $a_{i i}=1$ or $a_{i i}=-1$.

2. Second, fill in any random integers at each entry with $i<j$. Afterward, it has formed a top triangular matrix whose determinants are 1 or -1 . This is a unimodular matrix.

3. Third, to be a complete matrix, use elementary row operations (ERO) "add a row with multiply of another row."

Based on Lemma 2 above, the researchers will select the value of $a_{11}$ as 1 in step one, as it will be applied to the positive integer value in this case. The researchers will use the Bernoulli Map function in the second stage to convert it into integers between 0 and 255 . In the third step, the researchers are still using the Bernoulli Map function so that the researchers 
can apply "add one line by multiplying another row." The derivative Bernoulli Map function is expressed as real numbers between 0 and 1 . Then, the researchers change from real to an integer by selecting the first three digits after the decimal point and modulating with 256 (Delmi et al., 2020; Rosen, 2019).

In 1976, May was the first to present the logistics map (Devaney, 2018). Despite the fact that one of the chaotic functions looks to be straightforward. The recursive formula is

$$
x_{n+1}=r x_{n} \bmod 1 .
$$

One of the chaotic functions utilized in cryptography applications is the Bernoulli Map. This method creates rows of real numbers, which may then be utilized to create a keystream. The Bernoulli Map method, which was created to discover the keystream plant, was utilized to advance to the next phase by restricting the number of integers raised to eight. This algorithm creates real numbers sequence that must be transformed into integers with a range of 0 to 255 as a keystream. The method begins by luting the key rows generated from Equation (2.1). After these keys are multiplied by 10000 and rounded down (floor) to form an integer row, the key is mapped in a range between 0 and 255 (Maulida, 2018). Bernoulli Maps produce a random series of numbers with a dispersion between 0 and 1 when $r>1$. (0.1). There are several methods for constructing a row of integers with values ranging from 0 to 255 . One is to modulate the results by 256 using three first digits following the decimal point of $\mathrm{x}$ given by the Bernoulli Map. The first three digits after the decimal point were employed in this inquiry. This paper offers four algorithms, each of which uses a unimodular matrix as the key. The matrix is created using Bernoulli Maps. Algorithms 1 and 2 are used in the encryption and decryption process. The four algorithms adopted from (Pareek et al., 2006; Ye \& Ma, 2013) with modification can be seen in Table 1.

Table 1. The Four Algorithms The Researchers Propose

\begin{tabular}{|c|c|}
\hline Algorithm 1: Generating Bernoulli Map Sequence & Algorithm 1: Generating Unimodular Matrix (Key) \\
\hline 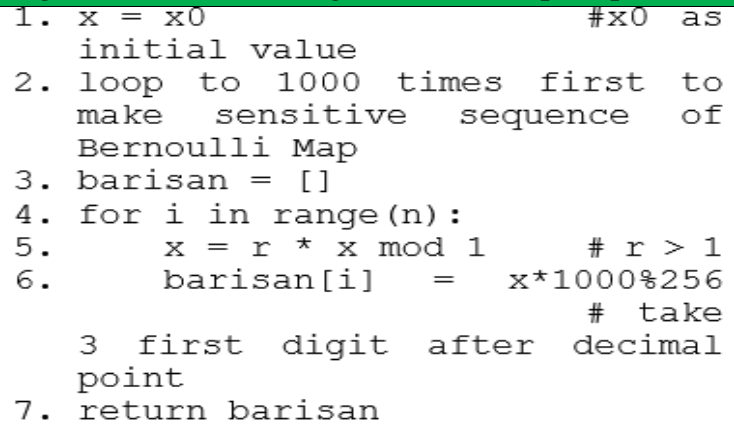 & 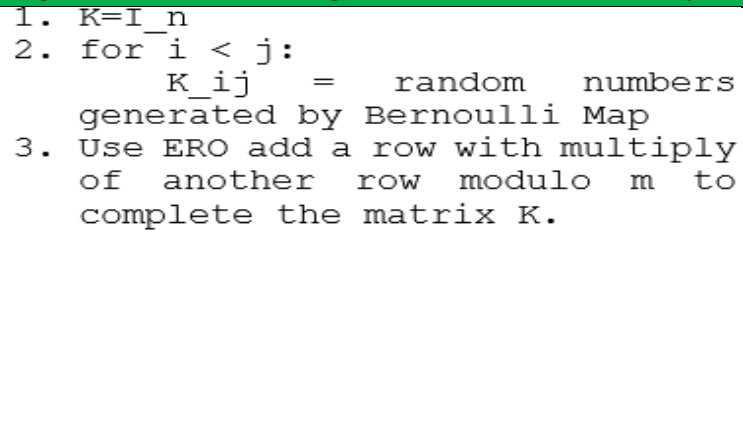 \\
\hline 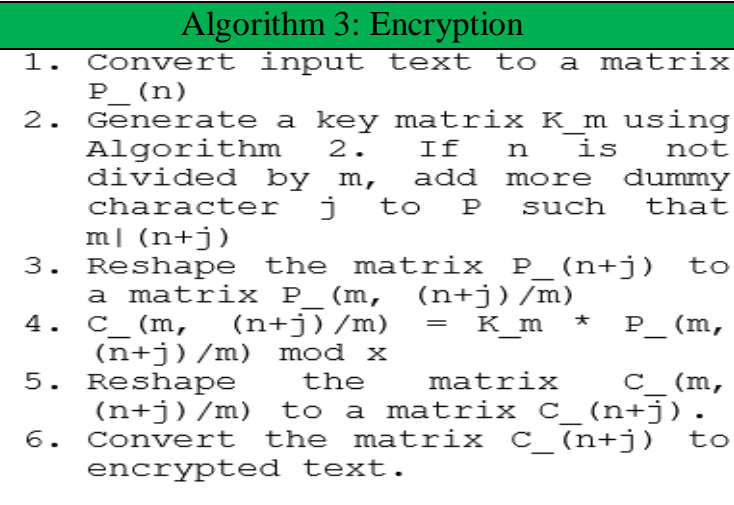 & 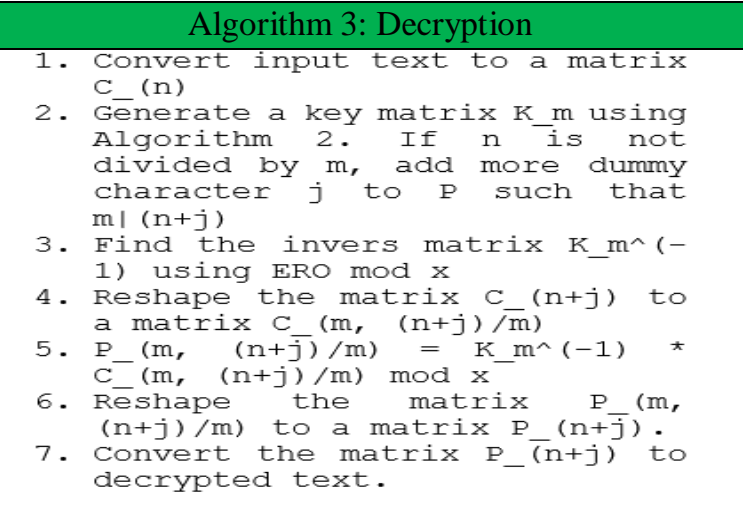 \\
\hline
\end{tabular}


If the researchers use the process in (Muktyas et al., 2021), one of the disadvantages is the existence of a keyspace for a small password 1, which is based only on the size of the image. As of this writing, password key 1 is expanded. This is still acceptable because the decrypted message reads like the original one. Look at the following example. Suppose the researchers want to encrypt a $P$ statement that reads as follows:

\section{kelompok kami berisi samsul arifin dan indra bayu muktyas}

In the sentence above, the researchers change first into Barisan or matrix line, which can be seen in Figure 1 below.

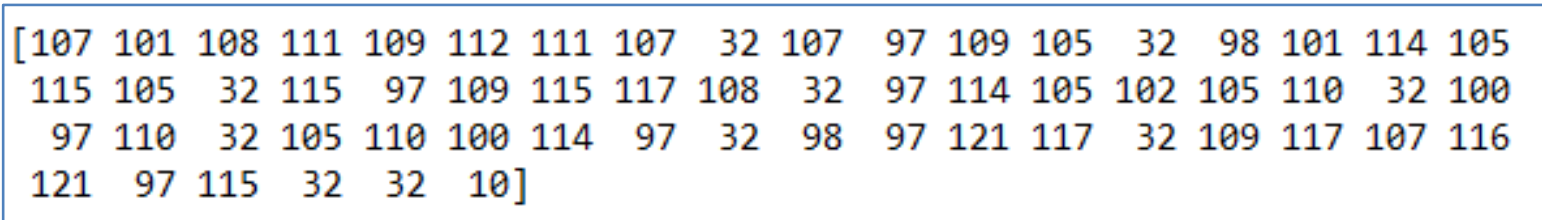

Figure 1. The Row Matrix P Corresponding to the Statement $P$

Note that the length of $P$ is 58 characters. Then the researchers add $P$ with the integer $n$ in such a way up to $5 \mid 58+n$. Note that in this case, the value of $n$ is 2 , and $58+2$ is 60 . As a result the set containing factors of 60 is $\{2,3,4,5,6,10,12,15,20,60,30\}$. Next, create a key matrix sized according to the user input, for example, 5. This is password 1. As a result, the key matrix built is a $5 x 5$ unimodular matrix, obtained from algorithm 2. Furthermore, suppose password 2 is 211101 , then based on the given 1 and 2 passwords, the generated $5 \times 5$ unimodular matrix can be seen in Figure 2.

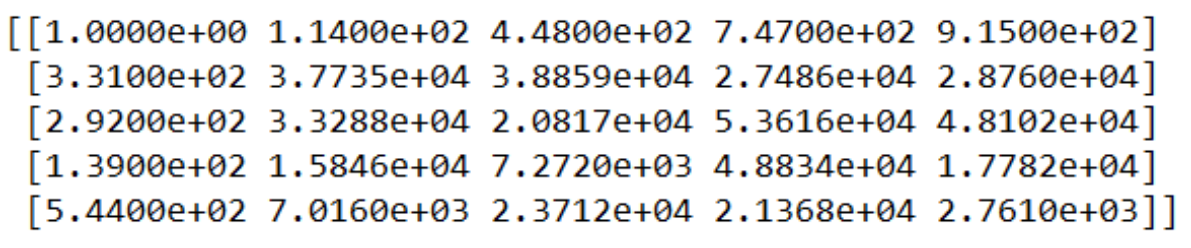

Figure 2. The Unimodular Matrix $\mathrm{K}_{5 \times 5}$ (Key Matrix)

Note that the size of the P matrix is adjusted to the $\mathrm{K}$ matrix so that it can be multiplied, bringing the size of the $\mathrm{P}$ matrix to $5 \times 12$. Next is to do the encryption process that multiplies the matrix of $K_{5 \times 5}$ and $P_{5 \times 12}$, which produces a matrix $C$ measuring $5 \times 12$, and if made into a matrix of rows, will be as follows: in Figure 3.

\begin{tabular}{|c|c|c|c|c|c|c|c|c|c|c|c|}
\hline [23111 & 2615 & 18303 & 46451 & 16636 & 23994 & 36134 & 53987 & 14849 & 54983 & 17230 & 46872 \\
\hline 32439 & 51234 & 9942 & 12414 & 17972 & 48933 & 54825 & 52072 & 25259 & 53380 & 52352 & 43500 \\
\hline 29125 & 22321 & 15826 & 28258 & 5583 & 12514 & 48883 & 6464 & 2787 & 17866 & 48420 & 5476 \\
\hline 37375 & 52699 & 24222 & 36643 & 11393 & 49518 & 34977 & 27199 & 42698 & 16839 & 49171 & 31299 \\
\hline 32501 & 47592 & 1941 & 24461 & 30091 & 17852 & 22017 & 54025 & 47971 & 45784 & 23152 & 33378] \\
\hline
\end{tabular}

Figure 3. Matrix $C_{5 \times 12}$, as the Product of the Matrix $K_{5 \times 5}$ and $P_{5 \times 12}$

The matrix $C_{5 \times 12}$ above indicates that the encryption process is complete. The next process is to decrypt, which is to multiply between the inverses of matrix $K$ and $C$. Note that the unimodular matrix used guarantees that every encryption process can be decrypted again because every unimodular matrix must have an inverse. 


\section{RESULTS AND DISCUSSION}

The researchers used Intel® Xeon® CPU E5-2650 v2 @2.60GHz $2.60 \mathrm{GHz}, \mathrm{RAM} 32 \mathrm{~GB}$, Windows 10 64-bit. The proposed algorithm was successfully implemented on text lorem ipsum. The Python program code can be seen in Figure 4.

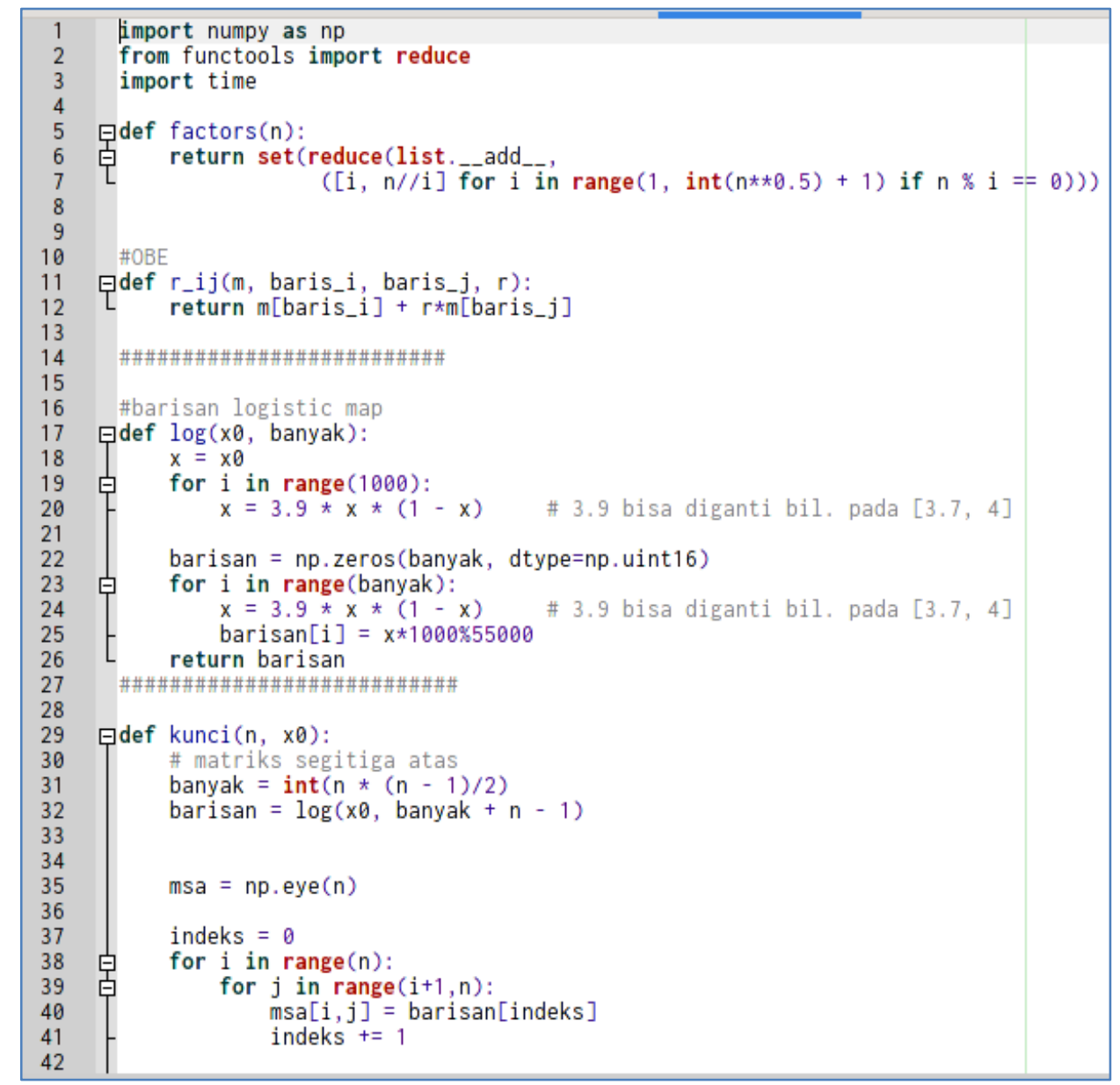

Figure 4. Python Code Display on Windows OS

Figure 5 contains the text we used as a test was dummy text taken from a famous dummy text that is usually used from lipsum.com site that contained five paragraphs, which is as follows:

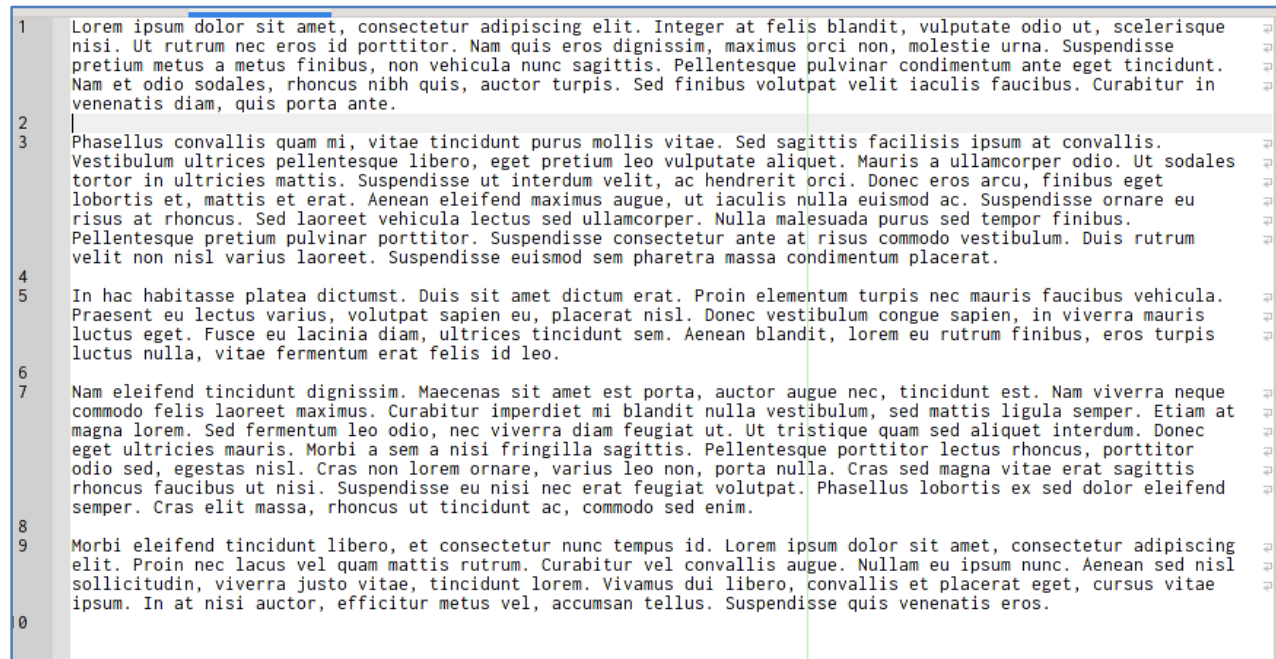

Figure 5. The Dummy Text 
Figure 6 is about the encryption result of the text with key 1: 10 and key 2: 22021985 as follows.

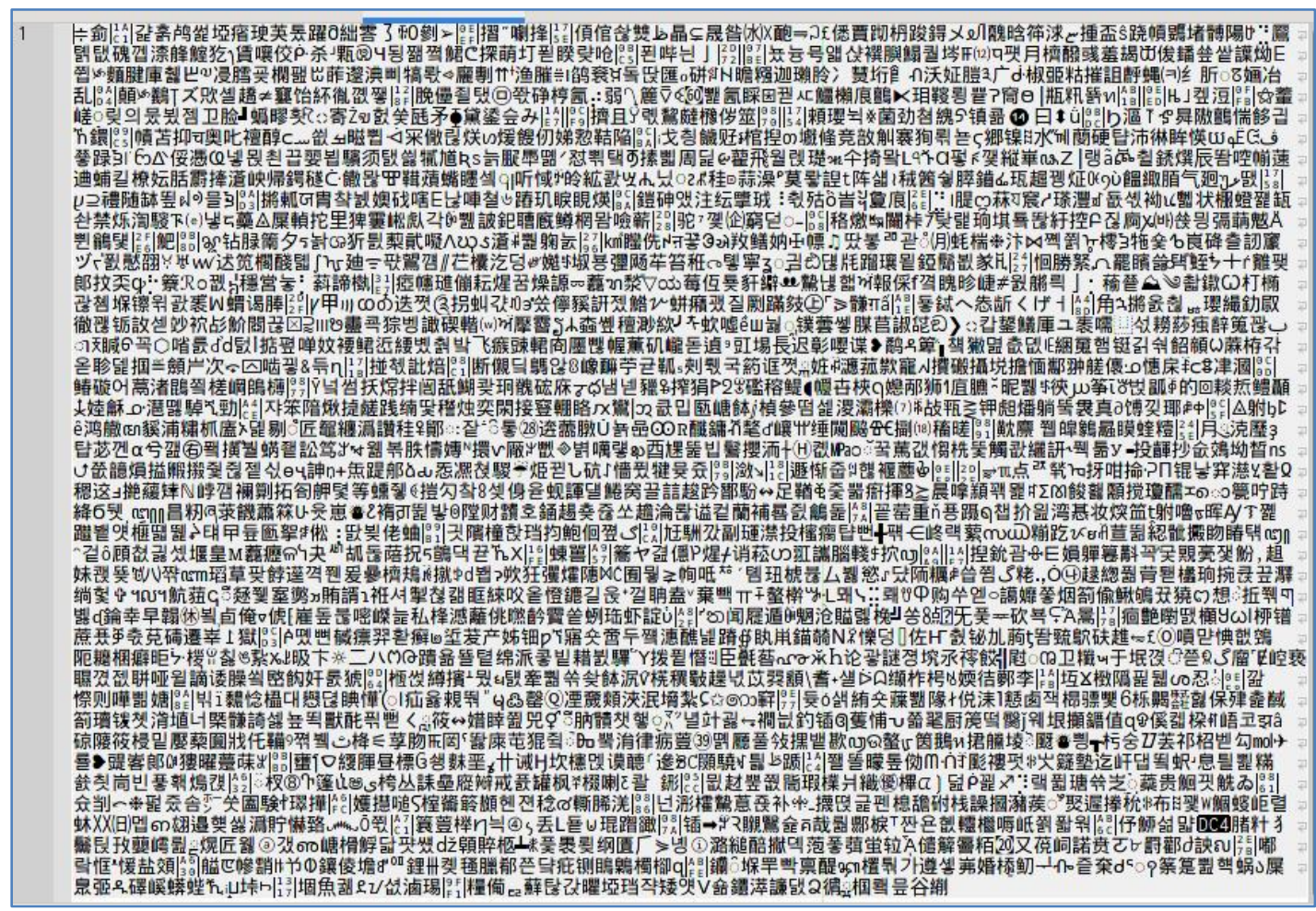

Figure 6. The Encryption Result

The characters are not well-read. It's good for encryption. Then after decrypting, the file is as follows in Figure 7. The decryption process can form back the ciphertext to be the plaintext, and the proposed algorithm guarantees this process

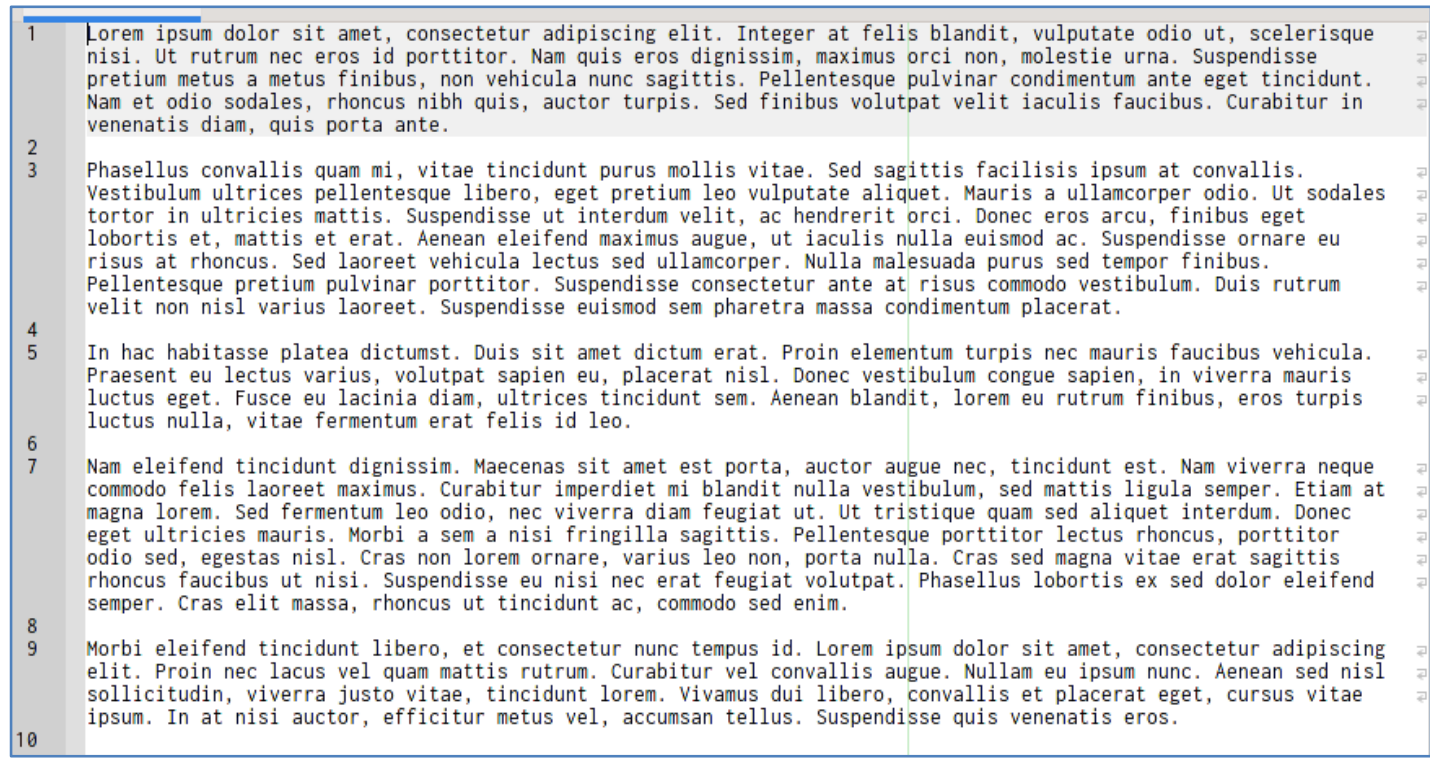

Figure 7. The Decryption Result

The full program code and example can be accessed at the following link: https://github.com/muktyas/text-encryption-chaos-unimodular. 
Research relevant to what the researchers do can be found in (Irsan \& Antoro, 2019). The objective of this research is to develop a method for encrypting text. By using the MS map as a keystream generator, the technique takes advantage of the chaotic map's characteristics. The proposed algorithm's ciphertext is extremely difficult to crack using bruteforce and known-plaintext attacks. The decryption process has been tested with three secret keys (all of which are extremely similar to the secret key used in the encrypted process). The decrypted text is identical to plaintext only if the secret input key is identical to that used in the encrypted process.

On the other hand, Krasimir Kordov's research is equally pertinent to our situation (Kordov, 2021). In this article, a new encryption technique for secure text message communication is given. The suggested cryptographic method is based on a pseudorandom generator that uses two chaotic maps to generate random numbers. An extensive cryptographic analysis is used to determine the security level. The findings of statistical testing, keyspace analysis, frequency analysis, common correlation analysis, entropy analysis, key sensitivity analysis, and speed performance are included in evaluating the proposed cryptographic system.

Hill Cipher is one of the methods used in cryptography in general. The square key matrix in the Hill Cipher algorithm must have an inverse modulo. The unimodular matrix is one of the unusual matrices that must have an inverse. A unimodular matrix can be utilized as a key in the encryption process. This unimodular matrix notion fills a research void because of its unique character, which is always invertible.

Additionally, the cryptography employed is symmetric cryptography. The approach is demonstrated by utilizing the Bernoulli Map function to generate a unimodular matrix. The researchers show an alternative to safeguarding text data.

\section{CONCLUSIONS}

The conventional Hill cipher uses a small key matrix $K_{n}, n=4$. Furthermore, if $n>4$, the entire matrix $K_{n}$ is used as the key using the usual Hill cipher. In this research, we use a Bernoulli Map as a key to building a Unimodular matrix that can solve the problem. $K_{n}$, yet only two arguments are required (password 1 and password 2). According to the results of the experiments based on the matrix property, the encrypted text messages tested were difficult to decipher in normal languages.

\section{ACKNOWLEDGMENT}

The researchers would like to express their gratitude to the reviewers for their insightful comments, recommendations, and ideas, which have helped shape this article into something that deserves to be published.

\section{AUTHOR CONTRIBUTIONS STATEMENT}

SA performs data acquisition, data analysis, compiling research manuscripts. IBM prepares the concept and design. PWP and AAA carry out supervision until final approval. 
Arifin, S., Muktyas, I. B., Prasetyo, P. W., \& Abdillah, A. A.

\section{REFERENCES}

Anton, H., \& Rorres, C. (2013). Elementary linear algebra: Applications version. John Wiley $\&$ Sons.

Arifin, S., Bayu Muktyas, I., \& Iswara Sukmawati, K. (2021). Product of two groups integers modulo m,n and their factor groups using python. Journal of Physics: Conference Series, 1778(1), 1-11.

Arifin, S., \& Muktyas, I. B. (2018). Membangkitkan suatu matriks unimodular dengan python. Jurnal Derivat: Jurnal Matematika Dan Pendidikan Matematika, 5(2), 1-10.

Arifin, S., \& Muktyas, I. B. (2021). Generate a system of linear equation through unimodular matrix using Python and Latex. AIP Conference Proceedings, 2331.

Delmi, A., Suryadi, S., \& Satria, Y. (2020). Digital image steganography by using edge adaptive based chaos cryptography. Journal of Physics: Conference Series, 1442(1), 17.

Devaney, R. (2018). An introduction to chaotic dynamical systems. CRC Press.

Guo, X., \& Yang, G. (2013). The probability of rectangular unimodular matrices over Fq[x]. Linear Algebra and Its Applications, 438(6), 2675-2682.

Hanson, R. (1982). Integer matrices whose inverses contain only integers. The Two-Year College Mathematics Journal, 13(1), 18-21.

Irsan, M. Y. T., \& Antoro, S. C. (2019). Text encryption algorithm based on chaotic map. Journal of Physics: Conference Series, 1341(6), 1-7.

Kordov, K. (2021). Text encryption algorithm for secure communication. International Journal of Applied Mathematics, 34(4), 705-719.

Maulida, S. (2018). Pengamanan citra biner dengan algoritma data encryption standard (DES) dan bernoulli map. Universitas Jember.

Muktyas, I. B., Sulistiawati, \& Arifin, S. (2021). Digital image encryption algorithm through unimodular matrix and logistic map using Python. AIP Conference Proceedings, 2331.

Nana, N., \& Prasetyo, P. W. (2021). An implementation of hill cipher and 3x3x3 rubik's cube to enhance communication security. Bulletin of Applied Mathematics and Mathematics Education, 1(2), 75-92.

Oliphant, T. E. (2007). Python for scientific computing. Computing in Science \& Engineering, 9(3), 10-20.

Pareek, N. K., Patidar, V., \& Sud, K. K. (2006). Image encryption using chaotic logistic map. Image and Vision Computing, 24(9), 926-934.

Rosen, K. H. (2019). Discrete mathematics and its applications (8th ed.). McGraw-Hill Education.

Siahaan, A. P. U. (2018). Application of hill cipher algorithm in securing text messages. International Journal For Innovative Research In Multidisciplinary Field, 4(10), 55-59.

W, S. (2006). Cryptography and network security: Principles and practices (4th ed.). Pearson Prentice Hall.

Ye, R., \& Ma, Y. (2013). A secure and robust image encryption scheme based on mixture of multiple generalized bernoulli shift maps and arnold maps. International Journal of Computer Network and Information Security, 5(7), 21-33. 